\section{Preliminary observations on avifauna of the Jai Prakash Narayan Bird Sanctuary (Suraha Tal Lake), Ballia, Uttar Pradesh, India}

\author{
P.K. Srivastava ${ }^{1} \&$ S.J. Srivastava ${ }^{2}$ \\ ${ }^{1,2}$ Department of Zoology, S.M.M. Town Post Graduate College, \\ Ballia, Uttar Pradesh 277001, India \\ Present address: ${ }^{1}$ Central Inland Fisheries Research Institute, \\ Barrackpore, Kolkata, West Bengal 700120, India \\ Email: ${ }^{1}$ pksrivastava17@yahoo.co.in (corresponding author), \\ 2shivajee1948@yahoo.co.in
}

Suraha Tal Lake is the largest floodplain lake in Ballia District of eastern Uttar Pradesh. It is an open type oval ' $U$ ' shaped ox-bow lake in the floodplain of river Ganga, located $8 \mathrm{~km}$ north of the district headquarters of Ballia. It is a perennial meander of the river Ganga with an area of $26 \mathrm{~km}^{2}$. During the monsoon season, it covers about $33.4 \mathrm{~km}^{2}$. It extends between $25^{\circ} 48^{\prime}-$ $25^{\circ} 52^{\prime} \mathrm{N}$ and $84^{\circ} 8^{\prime}-84^{\circ} 13^{\prime} \mathrm{E}$ at an altitude of $166 \mathrm{~m}$. The lake circumference is about $33.4 \mathrm{~km}$ (Image 1). The Government of Uttar Pradesh has notified an area of $34.4 \mathrm{~km}^{2}$ including the lake as a bird sanctuary by Gazette notification No. 1088(1)/14-3-19/89 Lucknow dated 24.03.1991. The sanctuary has been named "Jai

Date of publication (online): 26 July 2012

Date of publication (print): 26 July 2012

ISSN 0974-7907 (online) | 0974-7893 (print)

Editor: R. Jayapal

\section{Manuscript details:}

Ms \# 02042

Received 26 August 2008

Final received 16 July 2012

Finally accepted 17 July 2012

Citation: Srivastava, P.K. \& S.J. Srivastava (2012). Preliminary observations on avifauna of the Jai Prakash Narayan Bird Sanctuary (Suraha Ta Lake), Ballia, Uttar Pradesh, India. Journal of Threatened Taxa 4(7): $2727-2732$

Copyright: ( P.K. Srivastava \& S.J. Srivastava 2012. Creative Commons Attribution 3.0 Unported License. JoTT allows unrestricted use of this article in any medium for non-profit purposes, reproduction and distribution by providing adequate credit to the authors and the source of publication.

Acknowledgements: The authors are grateful to the Dr. A.K. Srivastava, Head, Department of Zoology, S.M.M. Town Post Graduate College, Ballia for their valuable suggestions during the entire study period. We are gratefu to anonymous reviewer for their thoughtful comments. We also wish to thank Dr. N.P. Shrivastava, Rtd. Principal Scientist and Joint Secretary, Inland Fisheries Society of India for necessary corrections.
Prakash Narayan Bird Sanctuary" and it comprises both private and Gram Samaj lands in a number

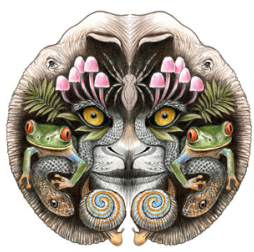
of small pokets where paddy is cultivated throughout the year. The lake is connected with the river Ganga through $32.6 \mathrm{~km}$ long Katehar nullah. The lake is drained and filled through Katehar nullah according to the water level of the river Ganga, resulting in complete inundation during the monsoon months. It offers good habitat for a variety of flora and fauna. Birds are known to arrive frequently this lake due to the availability of nesting and feeding habitats. The lake has great recreational value and supports local agriculture and tourism and also other activities common in low lying areas such as irrigation and fisheries. Human interference and alteration in water levels of the wetlands are significantly responsible to recent decline in bird population. So far there is no detail infomation on the population status of water birds and the possible impact of human activities on wild birds population of this region.

Materials and methods: The checklist of the avifauna of Suraha Tal Lake was prepared by extensive field surveys between August 2002 and July 2004. Surveys were conducted by fishing boat inside the entire lake and in paddy fields, trees and villages situated around the lake. Surveys were conducted monthly in the mornings from $0800-1100 \mathrm{hr}$ and in the evenings from 1500-1800 hr with the help of $8 \times 40$ Bushnell binoculars. Identification and records were maintained according to their status (resident, migrant and local migrant), season (summer, winter and throughout the year) and habitat (aquatic, trees and human habitation). Birds were identified with the help of books by King et al. (1975), Hancock (1984), Woodcock (1984), Ali \& Ripley (1987), Manakadan $\&$ Pittie (2001), and Ali (2002).

Results and Discussion: A total of 91 species of birds representing 33 families and 13 orders were recorded. Of these, 62 species are resident, 24 migrant and 20 are local migrant (Table 1). Availability of food and suitable habitat facilitated resident and local migrant bird species to visit the lake throughout the year (Fig. 1). In the winter season maximum birds species were recorded while in summer season the record was minimum. Common Teal, Temminck's Stint, Pallas's Fishing Eagle, and Great Crested Grebe 


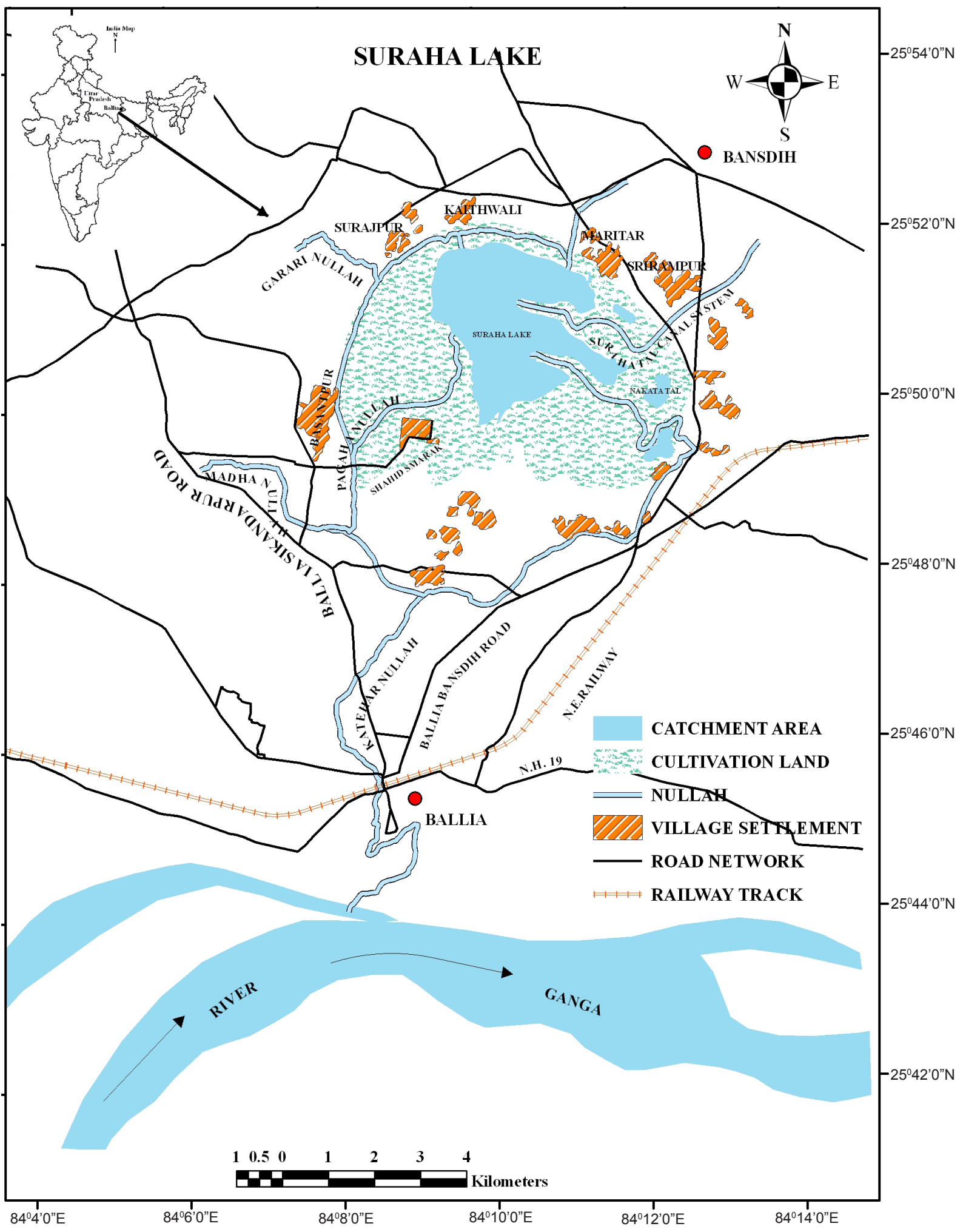

Image 1. Schematic map of Jai Prakash Narayan Bird Sanctuary (Suraha Tal Lake)

were recorded occasionally during the study.

The common resident birds were Grey Heron, Indian Pond Heron, Cattle Egret, Little Egret, Median Egret,
Great Cormorant, Indian Shag, Little Cormorant, Roseringed Parakeet, Greater Coucal, Indian Cuckoo, Indian Roller, Coppersmith Barbet, Brown-capped Pygmy 
Table 1. Checklist of birds recorded from Suraha Tal Lake area

\begin{tabular}{|c|c|c|c|c|c|c|}
\hline & Scientific name & Common name & Vernacular name & Status & Season & Habitation \\
\hline & $\begin{array}{l}\text { Ciconiformes } \\
\text { Ardeidae }\end{array}$ & & & & & \\
\hline 1 & Ardea alba & Large Egret & Bagula & $\mathrm{R}, \mathrm{LM}$ & W & $A$ \\
\hline 2 & Ardea cinerea & Grey Heron & Khanjan & $\mathrm{R}$ & W & $A$ \\
\hline 3 & Ardea purpurea & Purple Heron & Khyra & $\mathrm{R}, \mathrm{LM}$ & $\mathrm{T}$ & $A$ \\
\hline 4 & Ardeola grayii & Indian Pond Heron & Andha Bagula & $\mathrm{R}$ & $\mathrm{T}$ & $A$ \\
\hline 5 & Bubulcus ibis & Cattle Egret & Bagula & $\mathrm{R}$ & W & $A$ \\
\hline 6 & Egretta garzetta & Little Egret & Bagula & $\mathrm{R}$ & W & $A$ \\
\hline \multirow[t]{2}{*}{7} & Mesophoyx intermedia & Median Egret & Bagula & $\mathrm{R}$ & W & A \\
\hline & Threskiorhnithidae & & & & & \\
\hline 8 & Nycticorax nycticorax & Night Heron & Manduk & $\mathrm{R}, \mathrm{LM}$ & W & $A$ \\
\hline 9 & Pseudibis papillosa & Black Ibis & Kala Baz & LM & W & $A$ \\
\hline \multirow[t]{2}{*}{10} & Threskiornis melanocephalus & White Ibis & $\mathrm{Baz}$ & $\mathrm{R}, \mathrm{LM}$ & W & A \\
\hline & Ciconiidae & & & & & \\
\hline 11 & Anastomus oscitans & Open billed Stork & Ghonghil & $\mathrm{R}, \mathrm{LM}$ & W & A \\
\hline \multirow[t]{2}{*}{12} & Ciconia episcopus & White-necked Stork & Lag Lag & $\mathrm{R}, \mathrm{LM}$ & S & $A$ \\
\hline & $\begin{array}{l}\text { Anseriformes } \\
\text { Anatidae }\end{array}$ & & & & & \\
\hline 13 & Anas crecca (Linne) & Common Teal & Jal Murgi & M & W & A \\
\hline 14 & Nettapus coromandelianus & Cotton Teal & Girria & $\mathrm{R}, \mathrm{LM}$ & WS & A \\
\hline \multirow[t]{2}{*}{15} & Sarkidiornis melanotos & Comb Duck & Nakta & $\mathrm{R}$ & W & A \\
\hline & $\begin{array}{l}\text { Falconiformes } \\
\text { Accipitridae }\end{array}$ & & & & & \\
\hline 16 & Accipiter badius & Indian Shikra & Shikra & $\mathrm{R}$ & $\mathrm{T}$ & $\mathrm{T}$ \\
\hline 17 & Calidris temminckii & Temminck's Stint & Chhota Panlawa & M & W & A \\
\hline 18 & Charadrius dubius & Little ringed Plover & Titir & M & $\mathrm{T}$ & $A$ \\
\hline 19 & Elanus caeruleus & Black winged Kite & Kapassi & $\mathrm{R}$ & W & A \\
\hline 20 & Haliaeetus leucoryphus & Pallas's Fishing Eagle & Machhmmanga & $M$ & W & A \\
\hline 21 & Pluvialis apricaria & Golden plover & Batan & M & W & A \\
\hline \multirow[t]{2}{*}{22} & Vanellus cinereus & Grey headed Lapwig & Vuer chirae & M & W & A \\
\hline & $\begin{array}{l}\text { Charadriformes } \\
\text { Charadriidae }\end{array}$ & & & & & \\
\hline 23 & Tringa glareola & Spotted Sandpiper & Chupka & $M$ & W & A \\
\hline 24 & Tringa hypoleucos & Common Sandpiper & Merwa & M & W & A \\
\hline 25 & Tringa nebularia & Common Greenshank & Timtima & M & W & A \\
\hline \multirow[t]{2}{*}{26} & Tringa totanus & Common Redshank & Chhota Batan & $M$ & W & A \\
\hline & Recurvirostridae & & & & & \\
\hline 27 & Glareola lactea & Pratincole or Swallow Plover & Babuibattan & $M$ & WS & $\mathrm{T}$ \\
\hline \multirow[t]{2}{*}{28} & Himantopus himantopus & Black winged Stilt & Gozpaun & $\mathrm{M}$ & $\mathrm{T}$ & A \\
\hline & Laridae & & & & & \\
\hline 29 & Larus ridibundus & Black headed gull & Dhomra & M & W & A \\
\hline 30 & Larus brunnicephalus & Brown headed gull & Dhomra & $M$ & W & A \\
\hline \multirow[t]{2}{*}{31} & Sterna aurantia & River tern & Tehri & $\mathrm{R}$ & $\mathrm{T}$ & A \\
\hline & Jacanidae & & & & & \\
\hline 32 & Hydrophasianus chirurgus & Pheasant-tailed Jacana & Piho & $\mathrm{R}, \mathrm{LM}$ & $\mathrm{T}$ & A \\
\hline 33 & Metopidius indicus & Bronze-winged Jacana & Kattoi & $\mathrm{R}$ & $\mathrm{T}$ & $A$ \\
\hline
\end{tabular}




\begin{tabular}{|c|c|c|c|c|c|c|}
\hline & Scientific name & Common name & Vernacular name & Status & Season & Habitation \\
\hline & $\begin{array}{l}\text { Columbiformes } \\
\text { Columbidae }\end{array}$ & & & & & \\
\hline 34 & Columba livia & Blue Rock Pigeon & Kabutar & $\mathrm{R}$ & $\mathrm{T}$ & $\mathrm{T}$ \\
\hline 35 & Streptopelia decaocto & Eurasian Collared-dove & Panduk & $\mathrm{R}, \mathrm{LM}$ & $\mathrm{T}$ & $\mathrm{T}$ \\
\hline 36 & Streptopelia chinensis & Spotted Dove & Panduk & $\mathrm{R}, \mathrm{LM}$ & $\mathrm{T}$ & $\mathrm{T}$ \\
\hline \multirow[t]{2}{*}{37} & Treron phoenicoptera & Yellow-legged Green Pigeon & Harial & LM & W & $\mathrm{T}$ \\
\hline & $\begin{array}{l}\text { Pelecaniformes } \\
\text { Phalacrocoracidae }\end{array}$ & & & & & \\
\hline 38 & Anhinga melanogaster & Darter or Snake Bird & Pandubbi & M & W & $\mathrm{T}$ \\
\hline 39 & Phalacrocorax carbo & Great Cormorant & Pankawwa & $\mathrm{R}$ & W & $\mathrm{T}$ \\
\hline 40 & Phalacrocorax fuscicollis & Indian Shag & Kaul & $\mathrm{R}$ & W & $\mathrm{T}$ \\
\hline \multirow[t]{2}{*}{41} & Phalacrocorax niger & Little Cormorant & Pankawwa & $\mathrm{R}$ & W & $\mathrm{T}$ \\
\hline & $\begin{array}{l}\text { Psittaciformes } \\
\text { Psittacidae }\end{array}$ & & & & & \\
\hline \multirow[t]{2}{*}{42} & Psittacula krameri & Rose-ringed Parakeet & Tota & $\mathrm{R}$ & $\mathrm{T}$ & $\mathrm{T}$ \\
\hline & $\begin{array}{l}\text { Cuculiformes } \\
\text { Cuculidae }\end{array}$ & & & & & \\
\hline 43 & Centropus sinensis & Greater Coucal & Mohoka & $\mathrm{R}$ & W & $\mathrm{T}$ \\
\hline 44 & Cuculus micropterus & Indian Cuckoo & Papiha & $\mathrm{R}$ & W & $\mathrm{T}$ \\
\hline \multirow[t]{2}{*}{45} & Eudynamys scolopacea & Asian Koel & Koel & $\mathrm{R}, \mathrm{LM}$ & W & $\mathrm{T}$ \\
\hline & $\begin{array}{l}\text { Apodiformes } \\
\text { Apodidae }\end{array}$ & & & & & \\
\hline 46 & Apus affinis (Gray) & House Swift & Babila & $\mathrm{R}$ & W & A \\
\hline \multirow[t]{2}{*}{47} & Cypsiurus balasiensis & Asian Palm Swift & Telchatta & $\mathrm{R}$ & $\mathrm{T}$ & A \\
\hline & $\begin{array}{l}\text { Coraciiformes } \\
\text { Alcedinidae }\end{array}$ & & & & & \\
\hline 48 & Alcedo atthis & Small Blue Kingfisher & Chhota Kilkila & $\mathrm{R}$ & $\mathrm{T}$ & $A$ \\
\hline 49 & Ceryle rudis & Lesser Pied Kingfisher & Kilkila & $\mathrm{R}$ & $\mathrm{T}$ & A \\
\hline 50 & Halcyon smyrnensis & White-breasted Kingfisher & Kourilla & $\mathrm{R}$ & $\mathrm{T}$ & $\mathrm{T}$ \\
\hline \multirow[t]{2}{*}{51} & Pelargopsis capensis & Stork-billed Kingfisher & Badami Kourilla & $\mathrm{R}$ & $\mathrm{T}$ & $\mathrm{T}$ \\
\hline & Meropidae & & & & & \\
\hline 52 & Merops orientalis & Small Bee-eater & Patringia & $\mathrm{R}$ & $\mathrm{T}$ & $\mathrm{T}$ \\
\hline \multirow[t]{2}{*}{53} & Merops phillipinus & Blue-tailed Bee-eater & Bada Patringia & $\mathrm{R}, \mathrm{LM}$ & $\mathrm{T}$ & $\mathrm{T}$ \\
\hline & Coraciidae & & & & & \\
\hline \multirow[t]{2}{*}{54} & Coracias benghalensis & Indian Roller & Nilkanth & $\mathrm{R}$ & $\mathrm{T}$ & $\mathrm{T}$ \\
\hline & $\begin{array}{l}\text { Pioformes } \\
\text { Capitonidae }\end{array}$ & & & & & \\
\hline \multirow[t]{2}{*}{55} & Megalaima haemacephala & Coppersmith Barbet & Chotta Basanth & $\mathrm{R}$ & S & $\mathrm{T}$ \\
\hline & Picidae & & & & $\mathrm{T}$ & \\
\hline \multirow[t]{2}{*}{56} & Dendrocopos nanus & $\begin{array}{l}\text { Brown-capped Pygmy } \\
\text { Woodpecker }\end{array}$ & Kathphorwa & $\mathrm{R}$ & W & $\mathrm{T}$ \\
\hline & $\begin{array}{l}\text { Podicipediformes } \\
\text { Podicipedidae }\end{array}$ & & & & & \\
\hline 57 & Podiceps cristatus & Great Crested Grebe & Pandubbi & M & W & A \\
\hline \multirow[t]{2}{*}{58} & Tachybaptus ruficollis & Little Grebe & Pandubbi & M & W & A \\
\hline & $\begin{array}{l}\text { Passeriformes } \\
\text { Alaudidae }\end{array}$ & & & & & \\
\hline \multirow[t]{2}{*}{59} & Alauda gulgula & Eastern Skylark & Bharat & $\mathrm{R}$ & W & A \\
\hline & Hirundinidae & & & & & \\
\hline 60 & Hirundo smithii & Wire-tailed Swallow & Leishra & $\mathrm{R}, \mathrm{LM}$ & W & $\mathrm{T}$ \\
\hline 61 & Hirundo rustica & Common Swallow & Ababeel & M & w & $\mathrm{T}$ \\
\hline
\end{tabular}




\begin{tabular}{|c|c|c|c|c|c|c|}
\hline & Scientific name & Common name & Vernacular name & Status & Season & Habitation \\
\hline & Laniidae & & & & & \\
\hline \multirow[t]{2}{*}{62} & Lanius schach & Rufous backed Shrike & Kajala & M, LM & W & $\mathrm{T}$ \\
\hline & Campephagidae & & & & & $\mathrm{T}$ \\
\hline \multirow[t]{2}{*}{63} & Tephrodornis pondicerianus & Common Woodshrike & Tartituiya & $\mathrm{R}$ & $\mathrm{T}$ & $\mathrm{T}$ \\
\hline & Artamidae & & & & & \\
\hline \multirow[t]{2}{*}{64} & Artamus fuscus & Ashy Woodswallow & Ababeel & LM & $\mathrm{T}$ & $\mathrm{T}$ \\
\hline & Stumidae & & & & & \\
\hline 65 & Acridotheres fuscus & Jungle Myna & Junglee Myna & $\mathrm{R}, \mathrm{LM}$ & $\mathrm{T}$ & $\mathrm{T}$ \\
\hline 66 & Acridotheres ginginianus & Bank Myna & Myna & LM & $\mathrm{T}$ & $\mathrm{T}$ \\
\hline \multirow[t]{2}{*}{67} & Acridotheres tristis & Common Myna & Myna & $\mathrm{R}$ & $\mathrm{T}$ & $\mathrm{T}$ \\
\hline & Corvidae & & & & & \\
\hline 68 & Corvus splendens & House Crow & Deshi Kawwa & $\mathrm{R}$ & $\mathrm{T}$ & $\mathrm{T}$ \\
\hline \multirow[t]{2}{*}{69} & Corvus macrorhynchos & Jungle Crow & Dom Kawwa & $\mathrm{R}$ & $\mathrm{T}$ & $\mathrm{T}$ \\
\hline & Pycnonotidae & & & & & \\
\hline \multirow[t]{2}{*}{70} & Pycnonotus cafer & Red-vented Bulbul & Bulbul & $\mathrm{R}$ & $\mathrm{T}$ & $\mathrm{T}$ \\
\hline & Muscicapidae & & & & & \\
\hline 71 & Cisticola juncidis & Zitting Cisticola & Ghas Ki Phutki & $\mathrm{R}$ & $\mathrm{T}$ & A \\
\hline 72 & Copsychus saularis & Oriental Magpie Robin & Daiyar & $\mathrm{R}$ & $\mathrm{T}$ & $\mathrm{T}$ \\
\hline 73 & Erithacus svecicus & Bluethroat & Nilkanth & M & W & A \\
\hline 74 & Orthotomus sutorius & Common Tailorbird & Phutki & $\mathrm{R}$ & $\mathrm{T}$ & $\mathrm{T}$ \\
\hline 75 & Rhipidura albicollis & White-throated Fantail & Chakdil & $\mathrm{R}$ & $\mathrm{T}$ & $\mathrm{T}$ \\
\hline 76 & Saxicoloides fulicata & Indian Robin & Kalchhum & $\mathrm{R}$ & $\mathrm{T}$ & $\mathrm{H}$ \\
\hline 77 & Turdoides caudatus & Common Babbler & Satbhai & $\mathrm{R}$ & $\mathrm{T}$ & $\mathrm{T}$ \\
\hline \multirow[t]{2}{*}{78} & Turdoides striatus & Jungle Babbler & Satbhai & $\mathrm{R}$ & $\mathrm{T}$ & $\mathrm{T}$ \\
\hline & Sittidae & & & & & \\
\hline \multirow[t]{2}{*}{79} & Sitta castanea & Chestnut-bellied Nuthatch & Kath phorwa & $\mathrm{R}$ & $\mathrm{T}$ & $\mathrm{T}$ \\
\hline & Motacillidae & & & & & \\
\hline 80 & Anthus rufulus & Paddyfield Pipit & Charchari & $\mathrm{R}$ & $\mathrm{T}$ & A \\
\hline 81 & Motacilla alba & White Wagtail & Dhoban & M & W & A \\
\hline 82 & Motacilla flava & Yellow Wagtail & Pan Pilakh & M & W & A \\
\hline 83 & Motacilla maderaspatenis & Large Pied Wagtail & Khanjan & $\mathrm{R}$ & W & A \\
\hline 84 & Motacilla citreola & Yellow-headed Wagtail & Pani Ka Pilkya & M & W & $A$ \\
\hline \multirow[t]{2}{*}{85} & Motacilla cinerea & Grey Wagtail & Pani Ka Pilkya & M & W & A \\
\hline & Nectannidae & & & & & \\
\hline \multirow[t]{2}{*}{86} & Nectarinia asiatica & Purple Sunbird & Shakar khora & $\mathrm{R}$ & $\mathrm{T}$ & $\mathrm{T}$ \\
\hline & Ploceidae & & & & & \\
\hline 87 & Eremopterix grisea & Ashy-crowned Finch-Lark & Diyora & $\mathrm{R}, \mathrm{LM}$ & $\mathrm{T}$ & $A$ \\
\hline 88 & Ploceus phillippinus & Baya Weaver & Baya & $\mathrm{R}, \mathrm{LM}$ & $\mathrm{T}$ & $\mathrm{T}$ \\
\hline 89 & Passer domesticus & House Sparrow & Goraiya & $\mathrm{R}$ & $\mathrm{T}$ & $\mathrm{H}$ \\
\hline 90 & Petronia xanthocollis & Yellow-throated Sparrow & Jangalee Goraiya & $\mathrm{R}$ & $\mathrm{T}$ & $A$ \\
\hline 91 & Ploceus manyar & Streaked Weaver & Bamani Baya & $\mathrm{R}$ & $\mathrm{T}$ & A \\
\hline
\end{tabular}

Status: R - Resident; M - Migrant; LM - Local Migrant

Season: S - Summer; W - Winter; T - Throughout the year

Habitat: A - Aquatic (swampy, grass, and paddy fields); T - Trees (small and large); H - Human habitations 


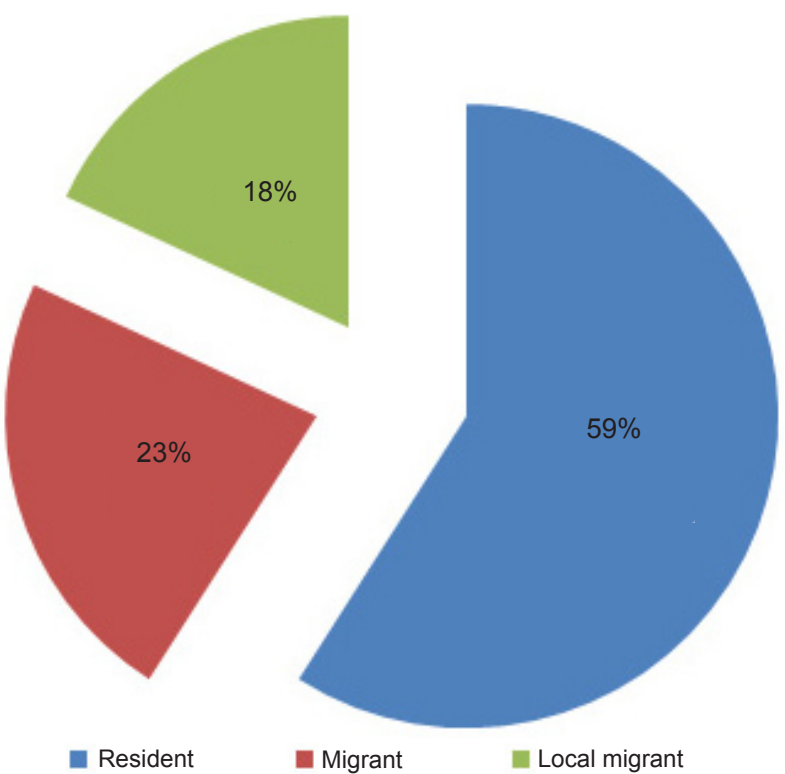

Figure 1. Percentage composition of avifauna of Suraha Tal Lake

Woodpecker, Common Myna, House Crow, Jungle Crow, Red-vented Bulbul, Jungle Babbler, Chestnutbellied Nuthatch and House Sparrow (Table 1).

The local migrant birds encountered were Large Egret, Purple Heron, Night Heron, Black Ibis, White Ibis, Baya Weaver, White-necked Stork, Cotton Teal, Pheasant-tailed Jacana, Eurasian Collared-dove, Spotted Dove, Yellow-legged Green Pigeon, Asian Koel, Blue-tailed Bee-eater, Wire-tailed Swallow, Rufous backed Shrike, Jungle Myna, Bank Myna, Ashy-crowned Finch-Lark and Open billed Stork (Table 1). The migratory birds mostly visited the area during winter season, were Common Teal, Little Ringed Plover, Golden Plover, Spotted Sandpiper and Darter are very common in appearance and also high in density.

The number of migratory birds has decreased over the years with increase in indiscriminate poaching. Another serious problem is the use of pesticides in paddy fields around the lake. A number of bird hunters kill the birds illegally either by trapping or poisoning. Poachers are adopting very special methods for birds hunting. They insert insecticides (Furadan) in the abdominal cavity of insects viz. (Forficula auricularia) and spread them near the vicinity of the lake and on the floating leaves of aquatic plants. Birds consume these poisoned insects, become lethargic and ultimately unconscious, and becoming easy prey to the poachers. The poachers revive them putting water drops in the bird's mouth. Then the live birds are furtively sold by them. Although the Forest Department has put up a signboard against the hunting of birds in these areas, they are still being hunted with the connivance of some local residents. Awareness programmes should be organized by local people, Government organizations and NGOs against the birds hunting and use of harmful pesticides in the agricultural fields. Human interference, eco-tourism and encroachment of wetlands are the main reasons for the decline in avifauna in terms of density as well as diversity.

\section{REFERENCES}

Ali, S. (2002). The Book of Indian Birds (13 $3^{\text {th }}$ Edition). Oxford University Press, New Delhi, 326pp.

Ali, S. \& S.D. Ripley (1987). Compact Handbook of Birds of India and Pakistan. Oxford University Press, New Delhi, 820pp.

Hancock, J. (1984). The Birds of The Wetlands. Oxford University Press, New Delhi, 176pp.

King, B., E.C. Dickinson \& M.W. Woodcock (1975). A Field Guide to The Birds of South-East Asia. Collins, London, 480pp.

Manakadan, R. \& A. Pittie (2001). Standardized common and scientific names of the Birds of the Indian Subcontinent BUCEROS. Envis news letter: Avian Ecology \& Inland Wetlands 6(1): 33pp.

Woodcock, M. (1984). Collins Hand Guide to The Birds of The Indian Sub-continent. Printed and bound by South China Printing Co. Hong Kong, 176pp. 